\title{
On-chip decoupling zone for package-stress reduction
}

\author{
Vincent L. Spiering ${ }^{\mathrm{a}}$, Siebe Bouwstra* and Ruud M.E J. Spiering ${ }^{\mathrm{b}}$ \\ ${ }^{\mathrm{B}}$ MESA Research Instrute and ${ }^{\mathrm{b}}$ Faculty of Mechanical Engineenng. Unversity of Twente, $P O$ Box 217, 7500 AE Enschede \\ (Netherlands)
}

(Received November 13, 1992, in revised form April 22, 1993, accepted April 27, 1993)

\begin{abstract}
A mathematical analysis is presented of the reduction of package stresses by introducing an on-chip decoupling zone Different configurations of the zones are compared, using the finte-element method (FEM) and analytical models A reduction of several orders of magnitude is obtained when a deep and thin axisymmetrical corrugation with a V-shaped cross section (a V-zone) is applied as the decoupling zone Approximate expressions for the stiffness and the force reduction are derved The application of a membrane pressure sensor with a V-zone as decoupling zone is evaluated It is shown that the sensitivity of the sensor is not reduced by the addition of the V-zone
\end{abstract}

\section{Introduction}

Many solid-state devices deal with the problem of package stresses $[1,2]$ To illustrate this problem, a membrane pressure sensor will be considered In this case the pressure is measured via the deformation of a thin pressure-loaded diaphragm in the sensor chip In general, the sensor chip is mounted on a backplate of a different matenal The thermal mismatch between the chip and the backplate causes stresses in the diaphragm, which fluctuate with ambient temperature and which disturb the sensor signal Figure 1 shows a scheme of a conventional packaged pressure sensor in a stressfree state, and in a deformed state due to shrinking of the backplate relative to the sensor chip Three

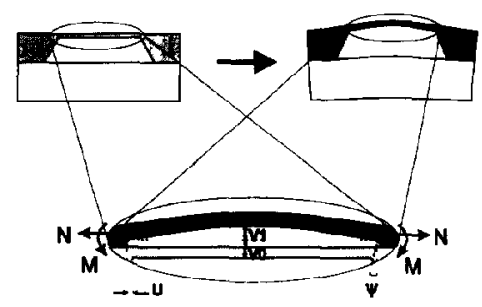

Fig 1 Deformations in a thin diaphragm of a conventional packaged pressure sensor due to shrinkage of the backplate relative to the sensor chip radial elongation $u$, accompanied by a planar force per unit length $N$ and a bending moment per unit length $M$, transverse displacement $v_{0}$ of the edge, and edge rotation $\psi$ (resulting in a centre deflection $v_{1}$ )

\footnotetext{
*Present address Microelectronics Centre (MIC), Lyngby, Denmark
}

different deformations occur in the diaphragm (1) a radial elongation $u$, accompanied by a planar force per unit length $N$ and a bending moment per unit length $M$, (2) a transverse displacement $v_{0}$ of the edge, and (3) a rotation $\psi$ of the edge (leading to a displacement $v_{1}$ of the centre relative to the edge) For the deformation of Fig 1 , the force $N$ and bending moment $M$ will be negative Depending on the sensor transduction mechanism, some of these deformations affect the output signal We assume that the vertical offset displacement $v_{0}$ does not affect the sensor output signal, which is true for strain-based applications like membranes with embedded strain gauges

Several methods to reduce package stresses have been proposed in the literature [2-6] To isolate the diaphragm from thermal and other package-induced stresses but to retain the sensitivity for stresses caused by a pressure difference, we propose to introduce a decoupling zone around the draphragm, see Fig 2 [7] The function of the decoupling zone is to transform

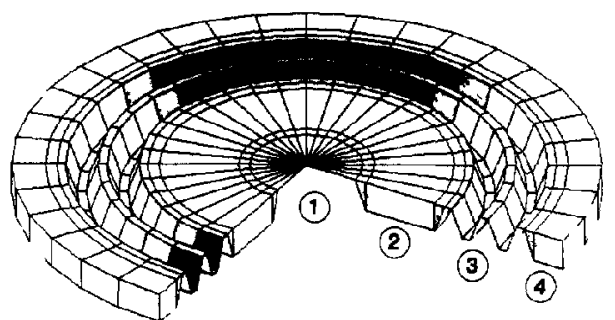

Fig 2 The sensor chip under investigation a sensor diaphragm (1) surrounded by an inner nm (2), a corrugated decoupling zone (3) and an outer rim (4) 
loads at the outer edge into local deformations with a small impact on the inner edge of the zone A circular corrugated zone serves this purpose very well, because a large tangential stiffness and a low radial stiffness are obtained simultaneously Moreover, in an axisymmetrical structure one type of stress concentration is avoided due to the absence of sharp corners in the plane of the zone Therefore, all structures considered here are axisymmetrical

The advantages of this concept are (1) a very large reduction due to the large tangential stiffness and the small radial stuffness of the curcular zone, (2) a good isolation, thanks to the fact that the decoupling zone directly encloses the sensor, (3) the bonding interface between the sensor chip and support is outside the decoupling zone, and (4) an additional intermediate is avoided Application is possible for transverse-loaded micromechanical devices, e g, pressure sensors, of which the sensor technology and zone technology will then have to be integrated

\section{Model}

For modelling the mechanical behaviour of the sensor chip, including the decoupling zone, the structure is divided into four parts, see F1g 2 (1) the diaphragm, (2) the inner rim, (3) the decoupling zone, and (4) the outer rim Before analysing the complete sensor chip, a theoretical model will be derived for three different configurations of the decoupling zone no decoupling zone, a flat decoupling zone, and a corrugated decoupling zone, see Fig 3 The corrugated zone consists of one corrugation with a V-shaped crosssection (a V-zone) With the stuffness of the decoupling zone small compared to the stiffness of the rums, it is practical to consider the zone as clamped at the inner radius and loaded by an imposed (mid-plane) displacement $u_{\text {out }}$ or rotation $\psi_{\text {out }}$ at the outer edge (Note by comparing the different decoupling zones, the same imposed displacements (rotations) are applied at the

(a)

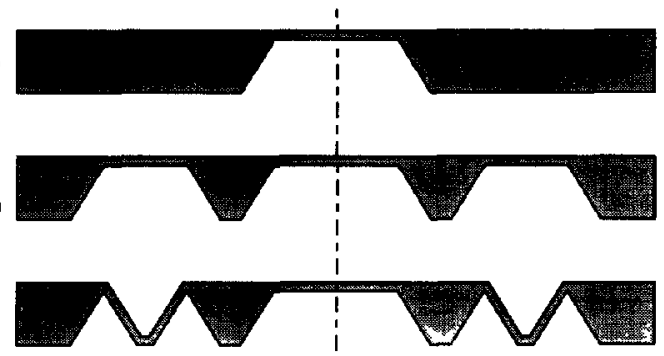

(c)

Fig 3 Cross sections of the different considered configuratıons (a) no decoupling zone, (b) flat decoupling zone, and (c) corrugated decoupling zone outer chip radius, which is allowed if the stiffness of the zone is small compared to its nm If the latter is not the case, then reducing the stiffness of the decoupling zone will result in an increase of the imposed displacement load ) The stiffness of the zone is represented by the stuffness matrix at the outer edge of the zone

$\left[\begin{array}{l}N_{\text {out }} \\ M_{\text {out }}\end{array}\right]=\left[\begin{array}{ll}S_{11} & S_{12} \\ S_{21} & S_{22}\end{array}\right]\left[\begin{array}{l}u_{\text {out }} \\ \psi_{\text {out }}\end{array}\right]_{u_{1 n} \psi_{\text {in }}-0}$

Note that according to Maxwell's reciprocal theorem [8], this stiffness matrix is symmetrical for linear elastic behaviour $\left(S_{12}=S_{21}\right)$

The reduction factor of the zone is defined as the ratıo between the internal forces or moments at the outer radius and inner radius

force reduction factor $=N_{\mathrm{out}} / N_{\mathrm{m}}$

moment reduction factor $=M_{\text {out }} / M_{\text {vn }}$

(see also Fig 4) Combinıng the stiffness matrix and the reduction factor, the zone can be characterized by a transfer matrix relating the force per unit length $N_{\text {in }}$ and moment per unit length $M_{\mathrm{in}}$ at the inner radius of the zone to the imposed (mid-plane) displacement $u_{\text {out }}$ and rotation $\psi_{\text {out }}$ at the outer radius of the zone

$\left[\begin{array}{l}N_{\text {1n }} \\ M_{\text {in }}\end{array}\right]=\left[\begin{array}{ll}T_{11} & T_{12} \\ T_{21} & T_{22}\end{array}\right]\left[\begin{array}{l}u_{\text {out }} \\ \psi_{\text {out }}\end{array}\right]_{\mu_{1 \mathrm{n}} \text { hn }=0}$

\section{The flat zone}

The flat zone is analysed as a circular plate with a circular hole at the centre, see Fig 4 The theoretical description of the flat zone is the same as for the structure without a zone The only difference is the value of the thickness $t$ (wafer thickness in the case of no zone) Expressions for the radial displacements $u(r)$ and the rotations $\psi(r)$ as a function of the planar force per unit length $N_{\text {uut }}$ and the bending moment per unit length $M_{\text {out }}$ are known from elementary mechanics for small displacements $[9,10]$ The force reduction factor and moment reduction factor are equal for the flat zone

$N_{\mathrm{ou} /} / N_{\mathrm{un}}=M_{\mathrm{out}} / M_{\mathrm{in}}=05\left[1+\nu+(1-\nu)\left(r_{\mathrm{in}} / r_{\mathrm{out}}\right)^{2}\right]$

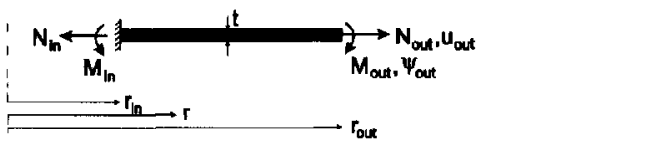

Fig 4 Definition of geometry and boundary conditions of the flat decoupling zone 
The value of this reduction factor will always lie between $(1+\nu) / 2$ and 1 It should be noted that the values of the forces or moments can be much larger in the casc of a thick zone due to the larger stuffness of this structure, leading to much higher stresses in the diaphragm For no flat zone, the actual imposed displacements/rotations at the outer edge will be smaller compared to a thin flat zone as a result of the increasing stuffness The transfer matrix of the flat zone becomes

$\left[\begin{array}{l}N_{\mathrm{in}} \\ M_{\mathrm{in}}\end{array}\right]=\frac{2 E r_{\text {out }}}{\left(1-\nu^{2}\right)\left(r_{\mathrm{out}}{ }^{2}-r_{\mathrm{in}}{ }^{2}\right)}\left[\begin{array}{ll}t & 0 \\ 0 & t^{3} / 12\end{array}\right]\left[\begin{array}{l}u_{\text {out }} \\ \psi_{\text {out }}\end{array}\right]$

with Young's modulus $E$, Poisson's ratio $\nu$, thickness $t$ and inner and outer radius $r_{1 \mathrm{n}}$ and $r_{\text {out }}$ respectively The cross-coefficients $T_{12}$ and $T_{21}$ in the matrox of eqn (3) are zero for a flat zone the displacement/force equations are not coupled with the rotation/moment equations Equation (5) shows that a smaller force $N_{\text {m }}$ and bending moment $M_{\mathrm{m}}$ are obtained for a smaller thickness $t$, a larger width $r_{\text {out }}-r_{\text {in }}$, and a larger muddle radius $\left(r_{\text {out }}+r_{\mathrm{in}}\right) / 2$ (as well as by a smaller Young's modulus)

\section{The circular V-zone}

\section{Analytıcal model}

For the analytical model the circular V-zone is separated into two conical shells which are connected at the bottom point The inner conical shell is defined as in Fig 5 with thickness $t$, inner radius $r_{\text {in }}$, outer radius $r_{\mathrm{m}}=\left(r_{\text {out }}+r_{\mathrm{m}}\right) / 2$, and angle $\gamma$, resulting in a depth $H=\left(\mathrm{r}_{\mathrm{m}}-r_{\mathrm{tn}}\right) \tan \gamma$ The solutions for the radial displacements $u(r)$, the rotations $\psi(r)$, the radial component of the internal forces per unit length $N(r)$ and the bending moments per unit length $M(r)$ as a function of the radial coordinate $r\left(r_{\mathrm{in}} \leqslant r \leqslant r_{\mathrm{m}}\right)$ are then [11]

$$
\begin{aligned}
u(r)= & \frac{t \cos \gamma}{4 \lambda}\left[A_{1}\left(2 \nu \operatorname{bel}_{2} x-x \operatorname{be}_{2}{ }^{\prime} x\right)\right. \\
& -\mathrm{A}_{2}\left(2 \nu \operatorname{ber}_{2} x-x \operatorname{ber}_{2}^{\prime} x\right) \\
& \left.+B_{1}\left(2 \nu \operatorname{kel}_{2} x-x \operatorname{kel}_{2}{ }^{\prime} x\right)-B_{2}\left(2 \nu \operatorname{ker}_{2} x-x \operatorname{ker}_{2}^{\prime} x\right)\right]
\end{aligned}
$$

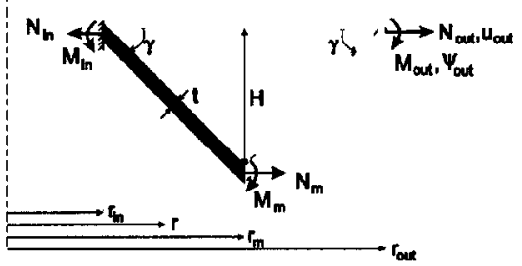

Fig 5 Definition of geometry and boundary conditions of the corrugated decoupling zone

$$
\begin{aligned}
N(r)= & \frac{-4 E t \sin \gamma}{x^{2} \cos ^{2} \gamma}\left[A_{1} \text { bel }_{2} x-A_{2} \text { ber }_{2} x\right. \\
& \left.\left.+B_{1} \operatorname{kel}_{2} x-B_{2} \operatorname{ker}_{2} x\right)\right] \\
\psi(r)= & A_{1} \operatorname{ber}_{2} x+A_{2} \operatorname{bel}_{2} x+B_{1} \operatorname{ker}_{2} x+B_{2} \operatorname{kel}_{2} x \\
M(r)= & \frac{E t^{2} \tan \gamma}{x^{2} \lambda}\left[A_{1}\left(2 \nu \operatorname{ber}_{2} x+x \operatorname{ber}_{2}{ }^{\prime} x\right)\right. \\
& +\mathrm{A}_{2}\left(2 \nu \operatorname{bel}_{2} x+x \operatorname{be}_{2}{ }^{\prime} x\right) \\
& \left.+B_{1}\left(2 \nu \operatorname{ker}_{2} x+x \operatorname{ker}_{2}{ }^{\prime} x\right)+\mathrm{B}_{2}\left(2 \nu \operatorname{kel}_{2} x+x \operatorname{kel}_{2}{ }^{\prime} x\right)\right]
\end{aligned}
$$

where

$\lambda=\left[3\left(1-\nu^{2}\right)\right]^{1 / 2}$

and

$x=(8 \lambda \tan \gamma / t \cos \gamma)^{1 / 2}$

The functions $\operatorname{ber}_{2}, \mathrm{bel}_{2}, \mathrm{ker}_{2}$ and $\mathrm{kel}_{2}$ are Kelvin functions of second order [12] These can be expressed in terms of Kelvin functions of zeroth order and their derivatives $[11,12]$ For large $x(x>25)$ the secondorder Kelvn functions can be approximated by

$$
\begin{aligned}
& \operatorname{ber}_{2} x \approx-\text { ber } x \approx-\frac{1}{(2 \pi x)^{1 / 2}} \cos \left(\frac{x}{2^{1 / 2}}-\frac{\pi}{8}\right) \exp \left(x / 2^{1 / 2}\right) \\
& \operatorname{be}_{2} x \approx-\text { beu } x \approx-\frac{1}{(2 \pi x)^{1 / 2}} \sin \left(\frac{x}{2^{1 / 2}}-\frac{\pi}{8}\right) \exp \left(x / 2^{1 / 2}\right) \\
& \operatorname{ker}_{2} x \approx-\operatorname{ker} x \approx-\left(\frac{\pi}{2 x}\right)^{1 / 2} \cos \left(\frac{x}{2^{1 / 2}}+\frac{\pi}{8}\right) \exp \left(-x / 2^{1 / 2}\right)
\end{aligned}
$$

and

$\mathrm{ke}_{2} \mathrm{x} \approx-\mathrm{kex} \approx\left(\frac{\pi}{2 x}\right)^{1 / 2} \sin \left(\frac{x}{2^{1 / 2}}+\frac{\pi}{8}\right) \exp \left(-x / 2^{1 / 2}\right)$

The constants $A$, and $B_{\imath}, l=1,2$, are to be determined from the boundary conditions The complete V-zone is modelled by connecting a second conical shell, with inner radius $r_{m}$, outer radius $r_{\text {out }}$, and the same top angle, but reversely orientated The eight constants $A_{i}$ and $B_{\imath}, \imath=1,2,3,4$, can be solved using the four boundary conditions at the edges (at $r=r_{\text {in }}$ and $r=r_{\text {out }}$ ) and the four equations of continuity of displacements and loads at the connection point (at $r=r_{m}$ ) In order to evaluate the $V$-zone, the inner radius is clamped for radial displacement $\left(u_{1 \mathrm{n}}=0\right)$ and rotation $\left(\psi_{1 \mathrm{n}}=0\right)$, as well as for a displacement in the axial direction In that case no transverse forces occur in the zone and the radial component of the internal force per unit length $N(r)$ is the total internal force per unt length in the V-zone At the outer radius the boundary conditıons are an imposed displacement $u=u_{\text {out }}$ and/or an imposed rotation $\psi=\psi_{\text {out }}$ Unless otherwise men- 
toned, a Young's modulus $E=150 \times 10^{11} \mathrm{~N} / \mathrm{m}^{2}$, a Porsson's ratio $\nu=017$ and a V-zone angle $\gamma=5474^{\circ}$ are applied

Figure 6 shows the normalızed displacements $u(r) /$ $u_{\text {out }}$ and the normalized internal forces $N(r) / N_{\text {out }}$ as a function of the radial coordinate in a V-zone under an imposed dispiacement $u_{\text {out }}$ of the outer radius $\left(\psi_{\text {out }}=0\right)$ These analytical results were verified by FEM calculations, using the Framatome software SYSTUS, with axisymmetrical shell-elements and differences were smaller than $1 \%$ (Note that by using these elements, unrealistic FEM results for the force at points close to the inner edge can be obtained because of problems with convergence) The discontinuity in the derivative of the radial displacement at the bottom of the $\mathrm{V}$ zone, as seen in Fig 6, was also observed in the FEM results The curves show the charactenstic behaviour of the V-zone described by the Kelvin functions a fast exponential decrease of effects at the outer edge, as well as an alternating nature

Approxamate reduction factor of forces and moments

From the above analytical model the reduction factors $N_{\text {out }} / N_{\text {in }}$ and $M_{\text {out }} / M_{\text {n }}$ can be determined Figure 7 shows the force reduction factor $N_{\text {out }} / N_{\mathrm{tn}}$ as a function of $K$, with $K$ defined as

$$
\begin{aligned}
K & =\left(x_{\text {out }}-x_{\mathrm{m}}\right) / 2^{1 / 2} \\
& =2\left[3\left(1-\nu^{2}\right)\right]^{1 / 4}\left[\tan ^{2} \gamma\left(1+\tan _{2} \gamma\right)\right]^{1 / 4} \frac{r_{\text {out }}{ }^{1 / 2}-r_{\text {in }}{ }^{1 / 2}}{t^{1 / 2}}
\end{aligned}
$$

The curves in $F_{1} g$ were plotted for fixed thickness $(t=2 \mu \mathrm{m}, H$ vanable) as well as for fixed depth $(H=200$ $\mu \mathrm{m}, t$ variable) for a range of values of $r_{\mathrm{in}}\left(r_{\mathrm{in}}=05\right.$, 15 and $25 \mathrm{~mm}$, respectively) and for $\gamma=5474^{\circ}$ For

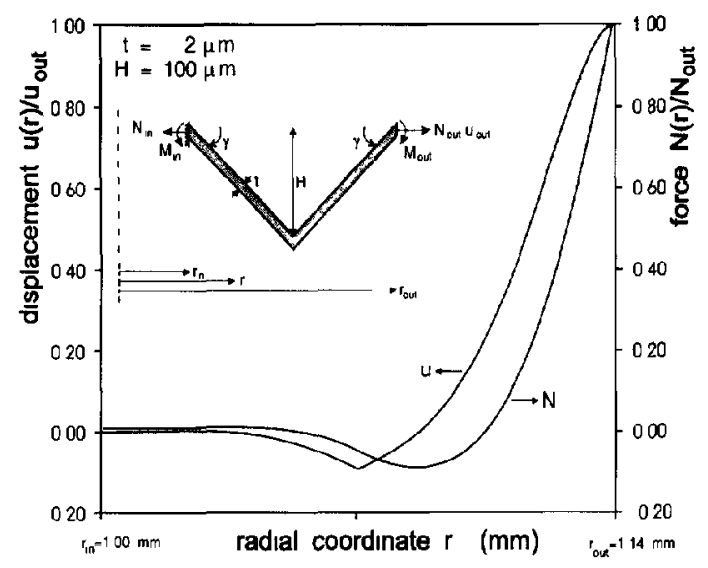

Fig 6 The normalized displacement $u / u_{\text {out }}$ and the normalized internal force $N / N_{\text {out }}$ as a function of the radial coordinate $r$ in a circular V-zone $H=100 \mu \mathrm{m}, t=2 \mu \mathrm{m}$ and $\gamma=5474^{\circ}$

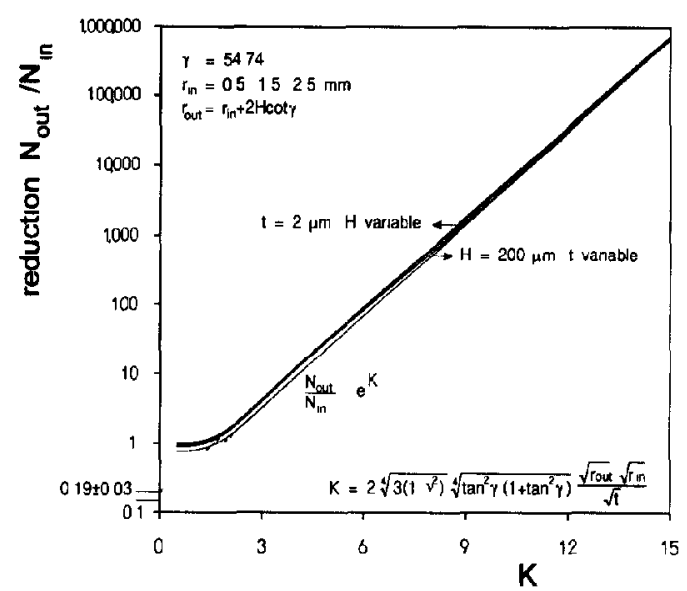

Fig 7 Analytical results for the force reduction factor $N_{\text {out }} / N_{\text {m }}$ as a function of $K$ for a circular V-zone The depth $H$ is varied for fixed thickness $t=2 \mu \mathrm{m}$ and the thickness $t$ is vaned for fixed depth $H=200 \mu \mathrm{m}$, both with $r_{\mathrm{tn}}=05,15$ and $25 \mathrm{~mm}$, respectively For all curves $\gamma=5474^{\circ}, E=150 \times 10^{11} \mathrm{~N} / \mathrm{m}^{2}$ and $\nu=017$

$K>2$ both reduction factors can be approximated by

$N_{\text {out }} / N_{\text {in }} \approx M_{\text {out }} / M_{\mathrm{in}} \approx C_{\text {red }} \exp (K)$

with $C_{\text {red }}=019 \pm 003$

The value of the proportionality factor $C_{\text {red }}$ was derived by linear extrapolation to $K=0$ For small values of $K$ ( $1 \mathrm{e}$, a thick and narrow zone) the exact solution, obtained using eqns (6), approaches a reduction factor of one, which is realistic For $K>2$ eqn (9) is very useful for estimating a reduction without making the laborious calculations described here For large $K$ the force $N_{\mathrm{in}}$ at the inner edge is very small Due to the alternating character of the Kelvin functions, it is even possible to obtain a zero or negative internal force $N_{\mathrm{m}}$ at $r=r_{\text {in }}$ while a positive force $N_{\text {out }}$ is acting at $r=r_{\text {out }}$ However, the required geometries $(\mathrm{e} g$, an extremely thin V-zone) are not realistic for this application This alternation was observed, but it is omitted in the representation of the results Equation (9) approaches the reduction factor within $2 \%$ for geometries for our purpose, but in general it represents the absolute lower bound value of the reduction factor for a certain $K$ The load used to obtain the results of Fig 7 was an imposed displacement, but an imposed rotation gives sumilar results The moment reduction factor $M_{\mathrm{out}} / M_{\mathrm{in}}$ also gives similar results for both load cases

\section{Approximate coeffictents of transfer matrx}

An approximate closed form was found for the four coefficients $T_{i j}$ of the transfer matrix by using relation (9) for the reduction factor and by deriving the elements of the stuffness matrix at $r=r_{\text {out }}$, see eqn (1) The approximate coefficients of the transfer matro of the V-zone become 


$$
\begin{aligned}
& {\left[\begin{array}{l}
N_{\mathrm{n}} \\
M_{\mathrm{m}}
\end{array}\right] \approx \frac{E}{C_{\mathrm{red}} \exp (K)}} \\
& \times\left[\begin{array}{ll}
\frac{t}{r_{\text {out }}\left(\frac{t}{r_{\text {out }} \lambda \sin \gamma}\right)^{1 / 2}} \frac{-t^{2}}{2 \lambda r_{\text {out }}} \\
\frac{-t^{2}}{2 \lambda r_{\text {out }}} & \frac{t}{2 \lambda}\left(\frac{t \sin \gamma}{\lambda r_{\text {out }}}\right)^{1 / 2}
\end{array}\right]\left[\begin{array}{l}
u_{\text {out }} \\
\psi_{\text {out }}
\end{array}\right]
\end{aligned}
$$

The coefficients $T_{12}$ and $T_{21}$ are not zero for this configuration $u_{\text {out }}$ not only causes a planar force per unit length $N_{\text {out }}$ but also a bending moment $M_{\text {out }}$, and so does $\psi_{\text {out }}$ The approximated transfer matrix is based on the stiffness matrux at the outer edge and is therefore symmetrical (within the accuracy of $C_{\text {red }}$ ) Because eqn (9) was used, the expressions for the coefficients are valid in the same range, $1 \mathrm{e}, K>2$ The approxumate solutions were verified with the exact analytical solutions To evaluate the latter, an equation solver software program was used

\section{Discussion}

Reduction factors of a V-zone with clamped inner edge and loaded outer edge are several orders of magnitude large Clamping the outer edge and loading the inner edge would result in a similarly large reduction factor If both edges are loaded, the influence of each load on the other edge is negligible the edges are decoupled It is not necessary to clamp one of the edges to require a large reduction Loading the outer edge with a free moving inner edge causes a large reduction of displacements and rotations Consequently the inner rim in the sensor chip is not essential for the reduction of stresses and it is possible to connect the V-zone directly to the diaphragm In that case the forces and bending moments, as well as the displacements and rotations, are reduced

FEM simulations showed that the maximum reduction factor for given values of width $r_{\text {out }}-r_{\text {in }}$ and angle $\gamma$ is always obtained by a minimum number of V-zones with maximum depth $H$ In the case of more than one $\mathrm{V}$-zone, the reduction factor is larger if all the $\mathrm{V}$-zones are directly connected, without rims in between An illustration is the choice between one single V-zone with depth $H$ or two equal V-zones with depth $H / 2$ To apply eqn (9) the assumption of a relatively stiff ring with zero radial length between both zones has to be made, so that both V-zones are clamped at their inner radius Equations (8) and (9) then show that the reduction factor of the single zone is about five times larger than the multiplication of the reduction factors of the two smaller V-zones For $n$ V-zones the total reduction factor then becomes

$$
\begin{aligned}
\left\{N_{\text {out }} / N_{\text {tn }}\right\}_{n \text { zones }} & =\left(C_{\text {red }}\right)^{n} \exp (K) \\
& =\left(C_{\text {red }}\right)^{n-1}\left\{N_{\text {out }} / N_{\text {tn }}\right\}_{1 \text { zone }}
\end{aligned}
$$

where $K$ refers to the single $V$-zone that would have a width of the total $n \mathrm{~V}$-zones and a depth of $n$ tumes the depth of one of the $n \mathrm{~V}$-zones

The results showed that the parameters of the $V$ zone with a major influence are the depth, the thickness and the width V-zones with different material properties or circular zones with alternative cross sections are expected to give similar results

\section{The sensor chip}

Two-dimensional FEM results are compared with analytical calculations of the whole sensor chip for the different configurations of the decoupling zone In this analysis the backplate is assumed to impose a radial displacement and a rotation at the outer edge of the sensor chip Again by companng the different decoupling zones the same imposed displacements (rotations) at the outer chip radius are applied, which is allowed if the stiffness of the chip is small compared to its housing If the relations between displacements, rotations, planar forces and moments for each part are known, then connection of the parts, applying the continuity of internal forces and of moments as well as the continuity of displacements and of rotations at the interfaces, yiclds the total solution Effects of local deformations at the interfaces are neglected, $1 \mathrm{e}$, displacements are due to transverse bending and planar elongation only Note that in the case of a step in the neutral plane, for example, between the diaphragm and the inner rim, there are two additional contributions at the interfaces the planar force of the connecting plate causes an additional contribution for the internal bending moments, and the rotation of the connecting plate causes an additional contribution for the radial displacements The rims are analysed as plates from which expressions for the radial displacements and the rotations as a function of the planar forces and the bending moments are known from elementary mechanics $[9,10]$

Figure 8 shows the resulting planar force in the sensor diaphragm when a radial displacement $u_{\text {out }}$ is imposed on the outer edge of the sensor chip The planar force was as good as uniform over the diaphragm in the different directions The diaphragm used for these calculations has a radius of $05 \mathrm{~mm}$ and thickness of $2 \mu \mathrm{m}$ The decoupling zones have the same thickness as the diaphragm and inner and outer radı of 15 and 


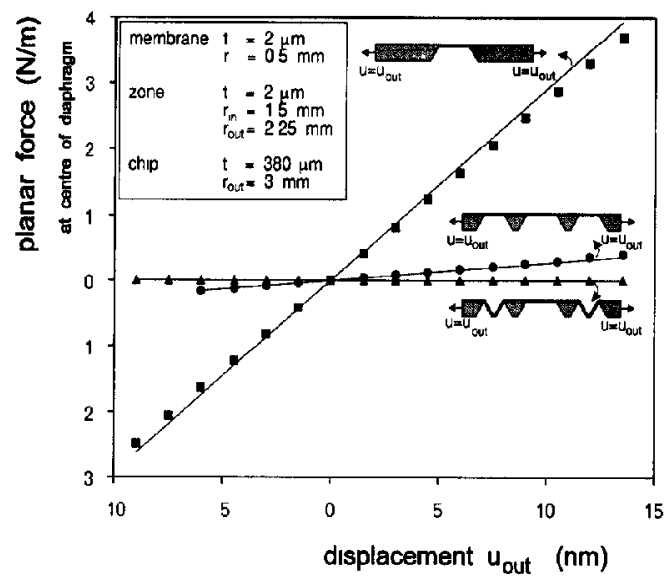

Fig 8 Analytical and FEM results for planar force in the diaphragm as a function of the imposed radial displacement $u_{\text {mit }}$ at the edge of the sensor chip for three different configurations

$225 \mathrm{~mm}$, respectively The corrugated decoupling zone contains a V-zone with a depth of $380 \mu \mathrm{m}$ The thickness and outer radius of the chip are $380 \mu \mathrm{m}$ and $3 \mathrm{~mm}$, respectively

The symbols represent the FEM calculations, using quadratic two-dimensional axisymmetric elements with non-linear large deflections For the considered displacement range, the linear analytical model (solid lines) shows a good agreement with the FEM results Only the configuration without a decoupling zone shows a hıgher-order deviation, due to non-linear effects not accounted for in the analytical model The reduction of the planar force in the diaphragm using a flat or corrugated decoupling zone is evident For a certain (negative) displacement $u_{\text {out }}$ buckling occurs in the flat decoupling zone, the slope of the curve beyond the buckling point, which was not included in these models, is expected to equal half the slope before buckling [13]

\section{Influence of anisotropy}

The influence of the anisotropic elastic properties of silicon compared to the (isotropic) analytical calculations was investigated Figure 9 shows Young's modulus $E$, Poisson's ratio $\nu$ and the value of $E /(1-\nu)$ and $E /\left(1-\nu^{2}\right)$ for silicon in a (100) plane [14] Depending on the geometry and kand of load, $E, E /(1-\nu)$ or $E /\left(1-\nu^{2}\right)$ represents an effective Young's modulus and in the case of biaxal loading $E /(1-\nu)$ should be used In the (100) plane the value $E /(1-\nu)=1805 \times 10^{11}$ $\mathrm{N} / \mathrm{m}^{2}$ is constant, while $E$ and $E /\left(1-\nu^{2}\right)$ vary from $130 \times 10^{11}$ to $169 \times 10^{11} \mathrm{~N} / \mathrm{m}^{2}$ and $141 \times 10^{11}$ to $170 \times 10^{11} \mathrm{~N} / \mathrm{m}^{2}$, respectively In all isotropic simulations and calculations Young's modulus $E=150 \times 10^{11} \mathrm{~N} / \mathrm{m}^{2}$ and Poisson's ratio $\nu=017$ were used, resulting in the value $1805 \times 10^{11} \mathrm{~N} / \mathrm{m}^{2}$ for $E /(1-\nu)$

In the case of braxial loading, like in-plane loading of a circular diaphragm, isotropic FEM simulations agreed with anisotropic FEM simulations in the (100) plane, which justifies the choice for $E$ and $\nu$ Also for a flat zone in a (100) plane (not purely biaxial loading) there was agreement to within $2 \%$ Due to the or1entation-dependent stuffness of the anisotropic V-zone, an increased overall stiffness of the zone, and therefore a decreasing reduction, is expected FEM simulations showed that the force reduction of the elastically anisotropic slicon V-zone was about $25 \%$ smaller in comparison with the elastically isotropic $V$-zone

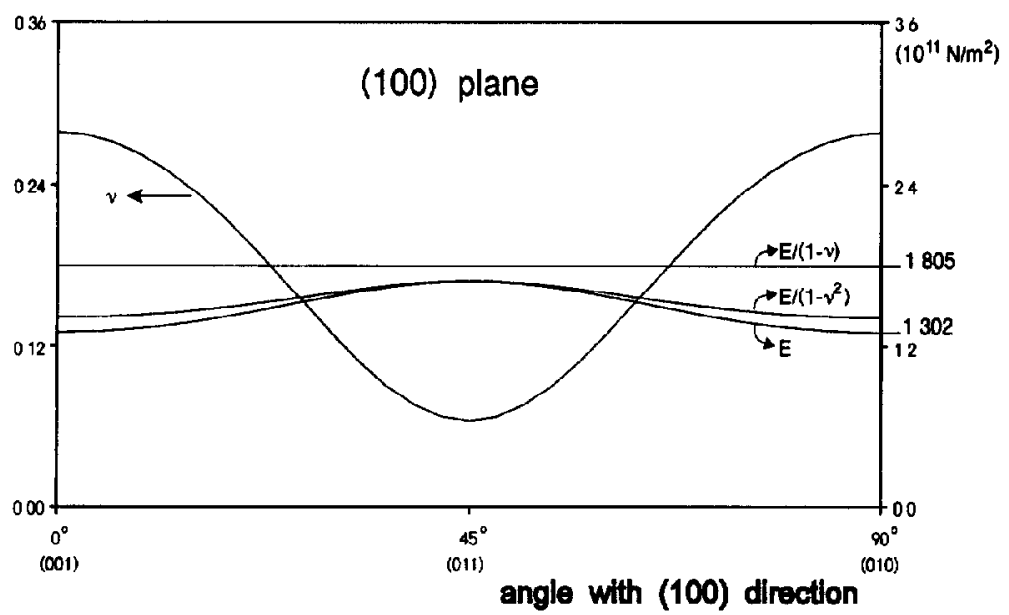

Fig 9 Young's modulus $E$, Poisson's ratio $\nu$ and the ratios $E /(1-\nu)$ and $E /\left(1-\nu^{2}\right)$ for silicon as a function of the direction in a (100) plane 
Influence of the decoupling zone on the sensitivity of the pressure sensor

FEM simulations to investigate the influence of the decoupling zone on the sensitivity of the pressure sensor were carried out The sensor chip in Fig 2, consisting of a thin diaphragm (1) and surrounded by a V-zone (3), is loaded by a pressure difference $\Delta P$ The function of the inner rim (2) is to maintain the edge condition for a clamped membrane, which means that the rotation at the edge remains zero We also investigated a structure with a decoupling zone without this inner nim The results are compared with a sensor chip containing a diaphragm only (with a wedge-shaped $\mathrm{rmm}$ ), with a clamped membrane and with a simply supported membrane The FEM results agreed with analytical results of the clamped and the simply supported membranes All configurations were simulated with zero initial strain (zero residual stress)

Figure 10 shows the strain in the upper fibre at the centre of the diaphragm as a function of the applied pressure difference for the different structures The dimensions of the diaphragm and zone are the same as used before, $1 \mathrm{e}$, for the diaphragm a thickness 2 $\mu \mathrm{m}$ and radius $05 \mathrm{~mm}$ and for the $\mathrm{V}$-zone the same thickness and a depth of $380 \mu \mathrm{m}$ The diaphragm with a wedge-shaped rum (without decoupling zone) shows small differences compared with the diaphragm with clamped edged, the difference being caused by the finite stiffness of the outer rim [15] The addition of the V-zone with thick inner rm $(380 \mu \mathrm{m})$ changes the strain in the upper fibre at the centre of the diaphragm by $1 \%$ or less This means that the requirement to

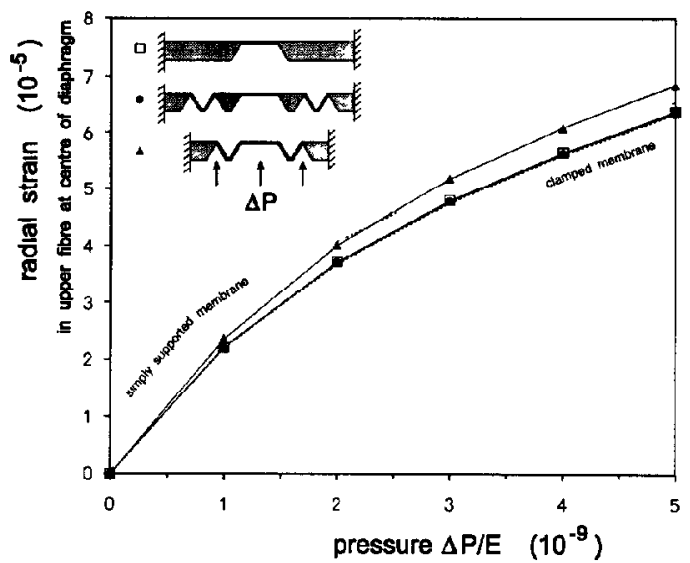

Fig 10 FEM results of the strain in the upper fibre at the centre of a pressure-loaded diaphragm for three configurations no decoupling zone, surrounded by $\mathrm{V}$-zone with inner rim, and surrounded by V-zone without inner nim Also shown are results for a membrane with clamped edge or simply supported edge All configurations are modelled for zero initial strain (zero resıdual stress) maintain the edge condition for a clamped membrane is achieved

The structure with the decoupling zone directly around the draphragm, $1 \mathrm{e}$, without inner rim, shows a slightly larger sensitivity of the strain in the upper fibres at the centre of the membrane compared to the first three structures with inner nm In fact the bending strain field in the whole diaphragm will change and a new design for the optımal sensitivity of this integrated decoupled membrane pressure sensor may be required In general the strain in the upper fibres at the centre of the diaphragm is not very sensitive for changes of the edge condition This result, combined with the large reduction and the efficient use of chip area, makes the device without inner nim very attractive for application in a membrane pressure sensor with strain gauges Besides, in this device without inner rim the initial strains and stresses are also reduced as a result of the stress relief appearing in the $V$-zone The other two structures will not have this advantage, and there an increasing initial strain will result in a decreasing pressure sensitivity

In this paper only the forces at the centre of the dıaphragm were evaluated The results are of interest for the application of decoupling zones in a strainbased differential pressure sensor The stress at the edge of the decoupling zone was not evaluated For a corner with infinite curvature the local stress would be infinite The actual stress very much depends on the actual shape at this position Obviously, infinite curvatures should be avoided in practice The actual shape at this position will not influence the global reduction of the decoupling zone

\section{Conclusions}

It was shown that it is possible to reduce package stresses by several orders of magnitude by introducing an on-chip decoupling zone around a device A flat zone and a corrugated zone, consisting of a circular shell with a V-shaped cross section, were mathematically analysed and the models were verified by means of FEM simulations This resulted in a design rule to describe the stress reduction due to the decoupling zone Theoretically, reduction factors of 1000 and higher can be realized by using a deep and thin axisymmetrical V-zone as decoupling zone On-chip application of the V-zone for a membrane pressure sensor based on strain measurement will not affect the sensitivity if the zone and the membrane are separated by a rum The onchip application with the V-zone directly connected to the edge of the diaphragm is attractive Besides the efficient use of the sensor chip, a large reduction of 
package-Induced stresses is achieved whlle the sensitivity of the sensor is slightly increased

\section{References}

1 S D Senturia and R L Smith, Microsensor packaging and system partitioning, Sensors and Actuators, 15 (1988) 221-234

2 V I Vaganov, Construction problems in sensors, Sensors and Actuators $A, 28$ (1991) 161-172

$3 \mathrm{H} \mathrm{L}$ Offerems and $\mathrm{H}$ Sandmaier, Novel stress free assembly technique for micromechanical devices, Proc Micro System Technol 90, Berlun, Germany, Sept 10-13, 1990, pp 515-520

4 B Halg and R S Popovic, How to liberate integrated sensors from encapsulation stress, Sensors and Actuators, A21-A23 (1990) 908-910

5 W Germer and G Kowalskı, Mechanical decoupling of monolithic pressure sensors in small plastic encapsulations, Sensors and Actuators, A21-A23 (1990) 1065-1069

6 H A C Tilmans, M Elwenspoek and J H J Fluitman, Micro resonant force gauges, Sensors and Actuators A, 30 (1992) 35-53

7 VL Spiering, S Bouwstra, R MEJ Spiening and M Elwenspoek, On-chip decoupling zone for package-stress reduction, Proc 6th Int Conf Solu-State Sensors and Actuators (Transducers '91), San Francisco, CA, USA, June 24-28, 1991, pp 982-985

$8 \mathrm{JM}$ Gere and S P Timoshenko, Mechanics of Matenals, Van Nostrand Reinhold, London, 2nd edn, 1988, pp 617-623

9 S Timoshenko and S Womowsky-Krieger, Theory of Plates and Shells, McGraw-Hill, New York, 2nd edn, 1970, pp $51-63$

10 S P Timoshenko and JN Goodier, Theory of Elasticity, McGraw-H1l, New York, 3rd edn, 1982, pp 65-80

11 F I Niordson, Shell Theory, North-Holland, Amsterdam, 1985, pp 272-281

$12 \mathrm{M}$ Abramowitz and I A Stegun, Handbook of Mathematical Functzons, Dover Publications, New York, 1970, Sections 99 and 910

13 W T Koiter, Lecture notes on 'mechanıcal stability', Unıversity of Delft, 1978

$14 \mathrm{~J} \mathrm{~J}$ Wortmans and R A Evans, Young's modulus, shear modulus and Poisson's ratio in silicon and germanum, $J$ Appl Phys, 36 (1965) 153-157

$15 \mathrm{~S}$ Bouwstra and B Geljselaers, On the resonance frequencies of microbridges, Proc 6th Int Conf Solud-State Sensors and Actuators (Transducers 91), San Francisco, CA, USA, June 24-28, 1991, pp $538-542$

\section{Biographies}

Vincent Sptenng was born in The Hague, The Netherlands, in 1965 He received his M Sc degree in applied physics from the Technical University of Twente, Enschede, The Netherlands, in 1989 His master's thesis was based on a plasmon sensor for selective protein detection In the same year he joined the MESA Research Institute at the University of Twente and worked with the finite-element method Currently he is workıng at MESA as a contract researcher on packaging of micromechanical sensors

Siebe Bouwstra was born in Amsterdam, The Netherlands, in $1958 \mathrm{He}$ received his M Sc degree in mechanical engineering from the Technical University of Twente, Enschede, The Netherlands, in 1984 In the same year he joined the Sensors and Actuators Research Unit (since 1990, MESA Research Institute) at the University of Twente as a contract researcher, where his research focused on micromechanical resonating structures In 1990 he received his $\mathrm{Ph} \mathrm{D}$ with a thesis based on a resonating microbridge mass-fiow sensor As a fellow of the Dutch Academy of Sciences, he worked for two years on surface micromachined resonatıng structures Currently he is working at the Micro Elcctronics Centre (MIC) at the Technical University of Denmark (DTH) in Lyngby

Ruud Splenng was born in Amsterdam, The Netherlands, in $1941 \mathrm{He}$ recelved his M Sc degree in mechanical engineering from the Technical University of Delft, The Netherlands, in 1966 His master's thesis was in the field of applied mechanics In the same year he joined the Department of Mechanical Engineernng at the University of Twente, Enschede, The Netherlands Currently he is working there as a semor scientist in applied mechanics and especially carries out research and provides student courses on finite-element methods 